\title{
CONSIDERAZIONI SULLA PRIMA CRISI IMPERIALE E LA CONDIZIONE DELL'INTELLETTUALE IN ETÀ NERONIANA
}

\author{
Carlotta Montagna* \\ University College of London, UK
}

Resumo. Sob Nero, a sociedade romana enfrentou sua Primeira Crise Imperial, consequência de um nível de poder e assistencialismo sem precedentes que se deveu sobretudo à falta de diretrizes políticas e culturais bem definidas após a morte de Augusto.

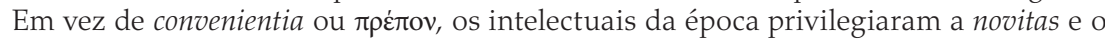
exibicionismo, criando assim a poética do impressionante. Quanto mais o poder imperial foi assumindo conotações absolutistas e despóticas, menos o papel e a contribuição social do intelectual pareceram dignos de consideração. Consequentemente, os intelectuais sugeriram um novo conceito de homem de letras, concentrando-se nos aspectos psicológicos do comportamento humano, como é o caso de Sêneca. A alternativa possível, conscientemente adotada por Lucano na Farsália, foi empenhar-se num gênero de poesia macabro e difamatório: a expressão de uma condição comum de fraqueza mental. Nesse sentido, as soluções estilísticas de Lucano são dignas de consideração: por meio de seu modus scribendi ardens e concitatus, Lucano expressou sua visão trágica da realidade e se desviou da tradição literária romana, que concedia pouco espaço para a irracionalidade. Sob Nero, houve uma renovação do interesse filosófico, mas já não parecia possível acreditar nas filosofias tradicionais. A persistência da crise levou os intelectuais de início a aceitar passivamente o status quo, mas depois passaram a se afastar dignamente da cena pública. Os hexâmetros de Pérsio nas Sátiras são emblemáticos a esse respeito.

Palavras-chave. Nero; crise; intelectual; Sêneca; Lucano; Pérsio.

D.O.I. 10.11606/issn.2358-3150.v17i1p34-41

L'ETÀ NERONIANA RAPPRESENTA UN PERIODO RICCO DI NUOVE PROSPETTIVE: a un susseguirsi di nuove conquiste territoriali ed esplorazioni geografiche ${ }^{1}$ si accompagna un generale miglioramento delle condizioni economiche.

Ciò nonostante, si fanno largo profonde incertezze interiori, si assiste a quella che propongo di chiamare la Prima Crisi Imperiale: l'inevitabile riflesso di una condizione di potere e benessere per l'appunto senza prece-

* PhD Student no University College of London. Classics.

** Artigo recebido em 21.out.2014 e aceito para publicação em 11.jan.2015.

${ }^{1}$ Sen. Nat. 6.8.3-4; Plin. Nat. 6.18.1. Le esplorazioni di Età Neroniana interessarono principalmente la regione caspica, 1'Etiopia e il nord Africa, alla ricerca della sorgente del fiume Nilo. 
denti (e in quanto tale destabilizzante) determinata dalla mancanza, da un lato, di chiare linee guida dinastiche e politiche dopo la morte di Augusto, dall'altra, di un progetto consapevole atto a proseguire la politica culturale augustea.

La res publica risulta ridotta a un mero sogno, a pura utopia per pochi nostalgici, se non, più semplicemente, a un argomento per esercizi scolastici di natura retorica: il regime augusteo è durato troppo a lungo perché persista una lucida consapevolezza dell'ideologia repubblicana. ${ }^{2}$ Condivisibile risulta pertanto la conclusione proposta dal Badalì: alla concreta restaurazione della repubblica non pensa seriamente più nessuno. ${ }^{3}$ Un profondo sentimento di caos, o sconforto, ha ora il sopravvento a fronte di una speranza pur diffusa nell'esercizio democratico e condiviso dell'interesse pubblico.

Da un punto di vista politico, il Senato si dimostra preoccupato, essenzialmente, per il potere personale assunto dal princeps, e dalla sua violenza tirannica, laddove la massa tende a considerare il potere monarchico come una sorta di freno all'arroganza senatoria. ${ }^{4}$

Per quanto concerne l'assetto socio-culturale, pare eloquente la reazione degli intellettuali al Principato, certo da connettere alla questione del patronato $^{5}$ : la morte di Mecenate, insieme alla conseguente interruzione di una saggia mediazione tra il potere politico e l'élite culturale, provoca tra l'anima politica e intellettuale dell'Impero un divario insanabile, se non in rare e sporadiche circostanze.

La restaurazione del mos maiorum assume la connotazione di una mossa meramente populistica e tra gli intellettuali si diffonde un crescente desiderio di chiusura e di ripiegamento in se stessi. Il delinearsi di una corrente storiografica di opposizione, che a partire dalla tradizione senatoria di età repubblicana si protrae almeno fino a Tacito e a Svetonio, appare al riguardo altamente significativo.

Si potrebbe obiettare che sotto Nerone Roma conosca il fenomeno di un Secondo Classicismo, finalizzato a un nuovo exploit letterario sulla scorta del paradigma augusteo. La mia opinione è che la sua portata non vada però troppo enfatizzata. Se ci fu, infatti, tale corrente classicistica si concluse in una breve esperienza, la cui esiguità risulta ampiamente confermata da opere mediocri come l'Iliade latina e le poesie bucoliche di Calpurnio Siculo, per non dire dall'affermazione di uno stile troppo barocco e magniloquente per essere paragonabile al misurato equilibrio del Classicismo augusteo.

\footnotetext{
${ }^{2}$ Bessone e Scuderi 2002, 251.

${ }^{3}$ Badalì 2007, 160.

${ }^{4}$ Pani 1972.

${ }^{5}$ Conte 1987, 305-6.
} 
In luogo di un ideale di convenientia, o пре́тov, appare preponderante un gusto per la novitas e l'esibizionismo o, per meglio dire, per quella che Cupaiolo ha definito la poetica dello straordinario. ${ }^{6}$

Ne consegue la ricerca dell'inaspettato e del paradossale, di risultati affettati, qualche volta poco ispirati. Chiara espressione di un crescente disagio interiore, i criteri estetici si fanno indeterminati e gli intellettuali si dimostrano spesso incapaci di nuove proposte artistiche credibili. Nel dettaglio, gli intellettuali romani procedono verso una riconsiderazione del valore degli antichi autori, con particolare attenzione rivolta a Virgilio, che viene in questo modo privato della sua fama di maggiore poeta della latinitas. ${ }^{7}$

Parallelamente, non senza il sostegno del princeps, si afferma un ideale di perfezione mai raggiunto in precedenza: quanto determina una parziale ridefinizione della gerarchia tra artes liberales e artes ludicrae avallata dalla tradizione, nonché l'apertura di apposite scuole per chi intenda esprimersi in pubblico. Tutto questo con l'intenzione di ampliare gli orizzonti del mondo sensibile e ultrasensibile conosciuto.

Nel frattempo, si diffonde il nuovo paradigma di un intellettuale lontano sia dal modello di poeta e vates di età repubblicana, sia dal sacerdos Musarum di Età Augustea.

Assistiamo a una progressiva chiusura dell'intellettuale in se stesso, a una progressiva riduzione, su un piano sociale, del suo ruolo e del suo contributo: maggiori sono le connotazioni assolutistiche e dispotiche del potere imperiale, minore risulta la considerazione del singolo.

Gli uomini di cultura scelgono pertanto di concentrare la propria attenzione su di sé, sui risvolti psicologici del comportamento umano, sulla propria solitudine esistenziale, e Seneca ne è l'emblematica conferma. Come sostenuto dal Bassi nel suo intervento datato, ma pur sempre valido, nel suo approccio al testo senecano il lettore di oggi finisce per credere di misurarsi con la pagina di uno psicologo moderno. Risulta in effetti difficile pensare che in Età Neroniana la capacità di osservazione fosse tanto sviluppata da cogliere certe sfumature e dettagli, da rivelare, in breve, un grado di sensibilità tuttora parzialmente insuperato. ${ }^{8}$

L'alternativa possibile, coscientemente adottata da Lucano nei Pharsalia, è l'adesione a una nuova sensibilità letteraria, la dedizione a un genere di poesia fatto di dettagli macabri e lascivi: la prova evidente di una comune condizione di debolezza psicologica, per non dire, persino, di una generale mancanza di equilibrio mentale.

\footnotetext{
${ }^{6}$ Cupaiolo 1994, 309.

${ }^{7}$ Lana 1990, 147.

${ }^{8}$ Bassi 1914, 50.
} 
Per circoscrivere le mie considerazioni al caso, pregnante, del Bellum Civile di Lucano, basti pensare, in merito, alla descrizione delle profezie dopo l'attraversamento del Rubicone da parte di Cesare, nel primo libro, ${ }^{9} \mathrm{o}$ ai versi dedicati alla guerra civile combattuta dagli eserciti di Mario e Silla e alla battaglia navale tra le flotte di Cesare e Pompeo, rispettivamente nei libri secondo e terzo. Significativa risulta anche la narrazione dello scontro di Farsalo e la descrizione dello spettacolo, desolante, che ne consegue, oltre all'episodio dei serpenti nel deserto libico, nel libro nono. Il pathos di Lucano raggiunge per altro il suo acme nel libro sesto, con la descrizione della maga Eritto e la scena di negromanzia di cui la maga è protagonista. ${ }^{10}$

Al di là dello specifico peso dei diversi episodi sopra indicati, ciò che colpisce è in realtà la coerenza stilistica di Lucano, la sua volontà di adottare lungo tutta la sua opera un modus scribendi ardens et concitatus, secondo la celebre definizione di Quintiliano. ${ }^{11}$ Lo stile diventa in questo modo l'espressione della visione del mondo di Lucano, del suo travaglio interiore, del quale il poeta stesso intende rendere partecipe il pubblico.

Le iuncturae lucanee, antitetiche e ossimoriche, a lungo studiate dal Fraenkel, affondano le loro radici nell'espressionismo e concettualismo paradossale della tarda letteratura repubblicana. ${ }^{12}$ Ciò nonostante, esse fanno dell'elemento insolito e inaspettato, del cosiddetto necopinatum, il leitmotiv e il protagonista indiscusso di tutta l'opera di Lucano, e questo è un dato senza precedenti. In merito, Badalì ha sostenuto che nei Pharsalia si avverte la prima emblematica comparsa del lato oscuro della psiche umana nella moderna letteratura. ${ }^{13}$ Si tratta di un'interpretazione senz'altro suggestiva, per quanto piuttosto iperbolica: metodologicamente più opportuno, per quanto più cauto, mi sembra il tentativo di rileggere l'opera di Lucano, e di valorizzarne gli elementi di novità, in una contesto culturale come quella romano, tendenzialmente ostile alle espressioni dell'irrazionale. Questo a riprova dell'entità della crisi culturale dalla quale l'Età Neroniana risulta pervasa.

Una condizione di tale disagio e sconcerto interiore può essere para-

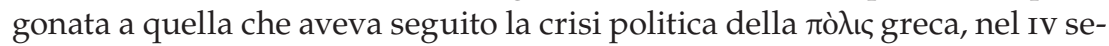
colo a.C. Allora, l'exploit di nuove correnti filosofiche, quella stoica ed epicurea in primis, aveva offerto una risposta esauriente alla diffusa richiesta di chiare indicazioni spirituali. ${ }^{14}$ Analogamente, in Età Neroniana, si assiste a

\footnotetext{
${ }^{9}$ Luc. $1.522-83$.

${ }^{10}$ Luc. 6.507-69.

${ }^{11}$ Quint. Inst. 10.1.90.

${ }_{12}$ Narducci 2002, 6.

${ }_{13}$ Badalì 2007, 162.

${ }^{14}$ Cupaiolo 1994, 281.
}

Let. Cláss., São Paulo, v.17, n. 1, p. 34-41, 2013 
un revival dell'interesse per gli studi filosofici, benché le tradizionali risposte offerte dalle scuole di pensiero ellenistiche risultino sostanzialmente insoddisfacenti. ${ }^{15}$ Segue, di conseguenza, la crisi del sistema stoico, culminante nella perdita di fiducia in una visione provvidenziale del mondo. $\mathrm{Ne}$ è prova il fatto che termini come fatum (positivamente connotato e da riferirsi a concetto di provvidenza) e fortuna (da intendersi nei termini di una sorte cieca), sebbene etimologicamente distinti, finiscano per confondersi e per identificarsi l'uno con l'altro. ${ }^{16}$

Gli intellettuali avvertono sempre più la crescente discrepanza tra la loro interiorità e la realtà circostante: avvertono la distanza da un mondo che non sentono loro ed è incapace non solo di riflettere la loro integrità morale, ma anche di assecondare, per lo meno, il loro desiderio di una rigenerazione etica del tessuto sociale. Le soluzioni adottabili, seppur in un persistente stato di inquietudine e di progressiva insoddisfazione, sono sostanzialmente due: l'accettazione più o meno passiva e formale dello status quo, per lo meno durante il quinquennium felix, da un alto, la deliberata condizione di polemico estraniamento dalle scene pubbliche, dall'altro lato. ${ }^{17}$

Altamente emblematici risuonano al riguardo gli esametri di Persio, dalla sua prima satira. Persio rivendica una differente sensibilità ed esprime la sua sincera intenzione di prendere le distanze dalla poesia contemporanea, la cui qualità gli sembra definitivamente compromessa dal deterioramento del gusto artistico e dal diffuso degrado morale. Soprattutto, Persio dà espressione alla sua esigenza di una considerazione effettiva per la figura dell'intellettuale. Ne seguono una critica puntuale del panorama intellettuale circostante e un affresco realistico della Roma di Nerone: una città dove, Persio ci dice,

ecce modo heroas sensus adferre videmus
nugari solitos graece, nec ponere lucum
artifices nec rus saturum laudare, ubi corbes
et focus et porci et fumosa Palilia faeno,
unde Remus sulcoque terens dentalia, Quinti
cum trepida ante boves dictatorem induit uxor
et tua aratra domum lictor tulit. ${ }^{18}$

Le parole di Persio sono velate di malinconia, come se la sua speranza in un ritorno a un passato irrimediabilmente perduto, in cui l'impegno degli intellettuali non era ancora ridotto a mero piacere per gli ospiti satolli

\footnotetext{
${ }^{15}$ Conte 1987, 281.

${ }_{16}$ Badalì 2007, 160-1.

${ }^{17}$ Conte 1987, 282.

${ }_{18}$ Pers. $1.70-6$.
} 
del banchetto, fosse destinata a infrangersi. ${ }^{19}$ Persio rimpiange il tempo in cui l'obiettivo ultimo dell'attività intellettuale non era un puro e generico elogio delle proprie capacità ${ }^{20}$ e la poesia non sorgeva dal desiderio di fama ${ }^{21}$ od omaggi $i^{22}$ o (quel che gli sembra persino peggio) dalla speranza di

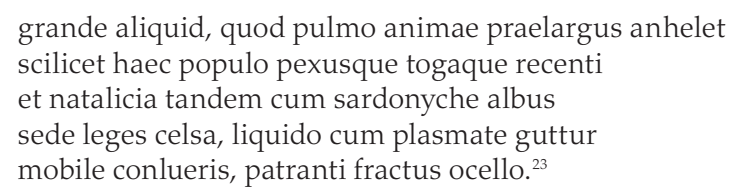

Che il tono e sentito rammarico di Persio muovano dalla severa considerazione dei vizi ampiamente diffuso nella società romane pare altamente probabile: Persio è condotto dalla sincerità della sua condanna a radere, defigere, revellere la realtà cittadina, nel tentativo di rigenerare la coscienza etica dei suoi concittadini, oltre che, ovviamente, la loro concezione di arte.

Persi sono ormai gli aurea saecula cantati da Virgilio, ${ }^{24}$ lontano è l'entusiasmo di Orazio per la riscoperta dei mores tradizionali nel Carmen Saeculare, ${ }^{25}$ lontana è, ormai, la preoccupazione di Orazio - "magna modis tenuare parvis" ${ }^{\prime 26}$ - nella celebrazione della potenza romana.

Una significativa testimonianza dello status quo emerge, ancora, dalle dichiarazioni di Persio e dalla sua orgogliosa affermazione di una condizione di alterità: di fronte alla corruzione, al vizio e al male dilagante in Roma, diventa ora per lui un motivo di merito e di onore sentirsi e definirsi semipaganus. ${ }^{27}$ Di fronte allo scenario della rovina morale di Roma, la soluzione adottata da Persio va interpretata come la significativa espressione di una precarietà esistenziale, che l'intellettuale può affrontare solo allontanandosi dalla realtà sociale, politica e culturale, sia fisicamente sia spiritualmente. ${ }^{28}$

Ho scelto di porre la mia attenzione, in particolare, sulla Satira 1, per il suo valore introduttivo, ma il discorso potrebbe essere facilmente esteso ad altri testi, soprattutto alla Satira 5, come suggerito dal La Penna, al quale rimando per ulteriori riflessioni. ${ }^{29}$

${ }^{19}$ Pers. $1.31-2$.

${ }^{20}$ Pers. $1.49-50$.

${ }^{21}$ Pers. 1.28 .

${ }^{22}$ Pers. $1.54-5$.

${ }_{23}^{23}$ Pers. 1, 15-19.

${ }^{24}$ Verg. A. 1.291, 6.792-3.

${ }^{25}$ Hor. Saec. 58-61.

${ }^{26}$ Hor. Carm. 3.3.72.

${ }^{27}$ Pers. pr.6.

${ }^{28}$ Mazzoli 2002, 164.

${ }^{29}$ La Penna 1979, 5-11. 
Mi chiedo se la pagina di Persio non meriterebbe una maggiore attenzione da parte della critica contemporanea, in una prospettiva storiografica.

Bellandi ha sottolineato la natura meramente etica dell'ottica dalla quale muove Persio. ${ }^{30}$ Ciò nonostante, credo che questo non consenta di escludere, alla base dell'opera di Persio, una profonda osservazione dell'attualità: se nella sua satira Persio finisce per agognare un passato utopico, credo, è perché egli muove dalla seria considerazione del presente, considerato inaccettabile.

La mia opinione è che Persio ci offra una vivida idea di quanto la Roma di Nerone dovesse essere culturalmente differente dal mondo augusteo, così profondamente connotato, in sostanza, da un sentimento di ottimismo per il futuro dell'Urbs e dell'Impero. Sotto Nerone, risulta infatti perduto l'entusiasmo che aveva guidato i maggiori autori legati al Circolo di Mecenate a celebrare Roma e i suoi protagonisti: più la memoria delle guerre civili si fa vaga, più il sistema etico basato sul mos maiorum e il concetto classico di ars sembrano vacillare. Questo nonostante la dichiarata intenzione di Nerone di seguire il nobile modello di Augusto, di cui Svetonio ci lascia esplicita testimonianza: "[Nero] ex Augusti praescripto imperaturum se professus [est]". ${ }^{31}$

\section{RIFERIMENTI BIBLIOGRAFICI}

Badalì, R. 2007. "Da Lucano a Stephen King: il nostro incubo quotidiano." In Atti del convegno nazionale di studi "Arma virumque cano". L'epica dei Greci e dei Romani, a cura di R. Uglione, 159-82. Torino.

Bassi, P. D. 1914. Seneca morale. Studi e saggi. Firenze.

Bellandi, F. 1988. Persio, dai "verba togae" al solipsismo stilistico: studi sui Choliambi e la poetica di Aulo Persio Flacco. Bologna: Pàtron.

Bessone, L. e R. Scuderi. 2002. Manuale di storia romana. Bologna.

Champlin, E. 2003. Nero. Cambridge, MA.

Cizek, E. 1986. La Roma di Nerone. Milano.

Conte, G. B. 1987. Letteratura latina. Firenze.

Cupaiolo, F. 1994. Storia della letteratura latina. Napoli.

Fraenkel, E. 1970. “Lucan als Mittler des antiken Pathos." In Vorträge der Bibliothek Warburg 1924-1925. Darmstadt.

Griffin, M. T. 1984. Nero: The end of a dynasty. London.

Lana, I. 1990. Sapere, lavoro e potere in Roma antica. Napoli.

\footnotetext{
${ }^{30}$ Bellandi 1988, 25 n. 10.

${ }^{31}$ Suet. Nero 10.1.
} 
La Penna, A. 1979. "Persio e le vie nuove della satira latina." In Persio: Satire, 5-78. Milan. Mazzoli, G. 2002. "La Roma di Persio." In Neronia VI: Rome à l'époque néronienne, édité par J.-M. Croisille et Y. Perrin, 161-9. Bruxelles.

Narducci, E. 2002. Lucano: un'epica contro l'impero. Firenze.

Pani, M. 1972. Roma e i re d'Oriente da Augusto a Tiberio. Bari.

*

Title. The First Imperial Crisis and the intellectuals in the Age of Nero

Abstract. Under Nero Roman society faced the First Imperial Crisis: the consequence of an unprecedented power and welfare, which was mainly due to the lack of clear po-

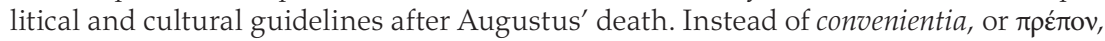
intellectuals privileged novitas and exhibitionism, thereby creating the poetics of the awesome. The more the imperial power went assuming absolutistic and despotic connotations, the less the intellectual's social role and contribution seemed to be worthy of consideration. Therefore, intellectuals suggested a new concept of the man of letters. They focused on the psychological aspects of the human behavior, as in Seneca's case. The possible alternative, consciously adopted by Lucan in Pharsalia, was to commit oneself to a macabre and slander gender of poetry: the expression of a common condition of mind weakness. In this regard, Lucan's stylistic solutions are worth of consideration: via his modus scribendi ardens and concitatus, Lucan expressed his tragic view of reality and diverted from the Roman literary tradition, which lent little space to irrationality. Under Nero there was a revival of philosophical interests, but the traditional philosophies looked not to be any more believable. The persistence of crisis led intellectuals first to passively accept the status quo, then to proudly estrange from the public scenes. With reference to this, Persius' hexameters in Satirae are highly emblematic.

Keywords. Nero; crisis; intellectual; Seneca; Lucan; Persius. 